\title{
Pharmacy Record Registration of Acetyl Salicylic Acid (ASA) Prescriptions in Quebec
}

\author{
Line Guénette ${ }^{1,2}$, Caroline Sirois ${ }^{3}$ \\ ${ }^{1}$ Faculty of Pharmacy, Laval University, Quebec, Canada. \\ ${ }^{2}$ URESP, Centre de recherche FRSQ du Centre hospitalier affilié universitaire de Québec, Quebec, Canada. \\ ${ }^{3}$ Division of Clinical Epidemiology, McGill University Health Centre, Montreal, Canada.
}

Received, February 1, 2012; Revised, March 22, 2012; Accepted, April 1, 2012; Published, April 2, 2012.

\begin{abstract}
Purpose: To determine the extent of which acetyl salicylic acid (ASA) use is included in patients' pharmacy records. Methods: During an in-home interview, people aged $\geq 65$ years were asked to report all of the medications they had used at least once, including over-the-counter drugs, during the preceding month. Researchers recorded information on the drug name, reason for its use, and whether a physician prescribed it. From the pharmacy records, the drug names, prescription fill dates, quantities supplied, and the numbers of days' supply were recorded. The level of agreement for ASA use across data sources was assessed using proportions of agreement and kappa coefficients. Results: Of 193 individuals interviewed, 86 reported the use of ASA, including 76 ASA users (88.4\%) who said it was prescribed by a physician. Pharmacy medication records indicated that there were 74 users of ASA. The proportion of agreement for ASA use was 93.8\%, and kappa coefficient was 0.87 (95\% confidence interval: 0.80-0.94). The sensitivity, specificity, and positive predictive value of the pharmacy data were all high. Conclusions: A large proportion of ASA use is documented in pharmacy records in Quebec. Thus, the information regarding ASA use in pharmacy records is reliable. This result may not be reproducible in other settings where pharmaceutical reimbursement rules are different.
\end{abstract}

This article is open to POST-PUBLICATION REVIEW. Registered readers (see "For Readers") may comment by clicking on ABSTRACT on the issue's contents page.

\section{INTRODUCTION}

Pharmacy records provide an excellent opportunity for studying drug use (1). In the province of Quebec, Canada, each prescription must be registered in pharmacy records, which ensures a representative picture of patients' prescription drugs profiles. Nonetheless, the main drawback of using pharmacy records is that the drugs available over the counter (OTC) are generally not registered and thus limits the evaluation of such drugs.

Acetyl salicylic acid (ASA) or aspirin is one of the rare drugs that is both widely prescribed (primarily for the prevention of cardiovascular events), and available OTC without prescription. Previous studies have suggested that nonprescription use of ASA may generate exposure misclassification bias when pharmacy data are used $(2,3)$. Because ASA is paid for when the drug is prescribed in Quebec, the individuals who take ASA on a regular basis may be more likely to fill their prescriptions for the drug than to buy ASA OTC. However, to our knowledge, no current data exist to confirm this assumption.
Therefore, the goal of our study was to determine the extent of which ASA use is included in pharmacy's patients profile.

\section{METHOD}

Data for this analysis were gathered using a cross-sectional study conducted among elderly individuals. During an in-home interview, participants had to report all drugs, including OTC drugs, that they had taken during the preceding month. Self-reported data were compared to pharmacy record information. The study design and methods have been fully described in two prior publications $(4,5)$. In brief, a convenient sample of 17 pharmacists, who all worked in different pharmacies in the Quebec City area, recruited the study participants. To minimize selection bias, individuals were recruited through random selection from a computerized list of patients who were at least 65

Corresponding Author: Line Guénette, 1050 Chemin Ste-

Foy, Québec (Qc) Canada;

Email address: line.guenette@pha.ulaval.ca 
years old. Individuals who had not been hospitalized in the last three months were asked to participate. A trained research assistant called all of the participants to provide them with detailed study information and to schedule an in-home interview. Participants were then informed that they should gather all of the medications they had used at least once in the preceding month, including OTC drugs, for the in-home interview.

This study was approved by the Centre hospitalier affilié universitaire de Québec's Ethics in Research Committee.

\section{Interviews}

Thorough interviews were conducted in participants' homes. Interviewers were blinded to participants' pharmacy information and used a structured computerized questionnaire.

We designed the interview to gather information on all of the drugs that each participant reported use during the preceding month. Information about drug name, dosage, pharmaceutical form, directions for use, and reasons for use was recorded. Participants were asked if each drug was prescribed by a physician or obtained OTC with this question "Has this medicine been prescribed by a physician?" Following each interview, a trained research assistant coded all of the drugs using the Anatomical Therapeutic Chemical (ATC) classification system (6).

\section{Pharmacy records}

The recruiting pharmacists supplied participants' pharmacy drug records for the one-year period prior to their interviews. We recorded drug names, directions for use, fill dates, quantities supplied, and the number of days' for the supply as they were entered in the pharmacy records. In addition, each drug was assigned its ATC code.

\section{Analysis}

We included ASA prescriptions that were extracted from pharmacy records and that had a treatment duration that overlapped with the 30 day observation window prior to participants' interviews. In addition, ASA use (prescription and non-prescription) were reported in the interview. We measured the proportion of agreement between the self-report and pharmacy record using a kappa coefficient and its $95 \%$ confidence interval (CI) (7). Finally, we calculated the sensitivity, specificity, and positive predictive value (PPV) of the pharmacy data (test) compared to those of the self-reports (gold standard). Selfreports were considered the gold standard as we aimed to test the validity of pharmacy data to measure the use of a medication also available over the counter. All analyses were conducted using SAS 9.1 (SAS Institute, Inc., Cary, North Carolina).

\section{RESULTS}

We compared information on ASA use from selfreports and pharmacy medication records for 193 individuals. Of these individuals, 86 (44.6\%) reported the use of ASA. Pharmacy medication records identified 74 users of ASA during an observation window corresponding to the 30-day period prior to participants' interviews (Table 1). The proportion of agreement for ASA use was $93.8 \%$ and the kappa coefficient was almost perfect (8) with 0.87 (95\% CI: 0.80-0.94). The sensitivity, specificity, and PPV for the pharmacy records were high (Table 1).

Among the 86 individuals who reported ASA use, 76 participants $(88.4 \%)$ said the drug was prescribed by a physician and 8 individuals (9.3\%) said they used ASA without a prescription. This information was missing for 2 participants. All but two of those who reported prescribed ASA mentioned cardiovascular reasons (i.e., to thin the blood or prevent a myocardial infarction). Two out of the 8 individuals who reported ASA use without a prescription said they used the drug for cardiovascular reasons. The 6 remaining participants said that they used ASA to help with pain $(n=4)$ or with colds $(n=2)$. The 2 ASA users for whom prescription drug information was missing said they used ASA for cardiovascular reasons (to thin the blood).

\begin{tabular}{|c|c|c|c|c|c|c|c|c|c|c|}
\hline \multirow[t]{2}{*}{ Pharmacy Records } & \multicolumn{2}{|c|}{ Self-reports } & \multicolumn{2}{|c|}{ Sensitivity } & \multicolumn{2}{|c|}{ Specificity } & \multicolumn{2}{|l|}{ PPV } & \multicolumn{2}{|c|}{ Kappa Coefficient } \\
\hline & Users & Non-users & $\%$ & $95 \% \mathrm{CI}$ & $\%$ & $95 \% \mathrm{CI}$ & $\%$ & $95 \% \mathrm{CI}$ & $\%$ & $95 \% \mathrm{CI}$ \\
\hline Users & 74 & 0 & & & & & & & & \\
\hline Non-users & 12 & 107 & & & & & & & & \\
\hline Total & 86 & 107 & 86.1 & $(77.2-91.8)$ & 100 & $(96.5-100)$ & 100 & $(94.8-100)$ & 0.87 & $0.80-0.94$ \\
\hline
\end{tabular}




\section{DISCUSSION}

Our results suggest that a high proportion of elderly patients residing in Quebec, Canada, have their ASA prescription filled in pharmacies when this antithrombotic agent is required for cardiovascular protection. The number of individuals who used non-prescribed ASA was low in this sample of older patients and use was usually related to non-chronic ailments.

Only a small proportion of the individuals included in our study used OTC ASA, which contrasts with previous studies' findings $(2,3)$. Delaney et al. studied a cohort of individuals aged 45-84 years old without a history of cardiovascular diseases and showed that among the 1562 patients who took ASA during the study period, 435 patients had a prescription for the drug, and 1127 patients used OTC ASA (2). Similarly, Ilkhanoff et al. indicated that the number of self-reported ASA users was higher than the number of individuals identified as ASA users by studies using electronic databases; researchers assumed that OTC ASA use probably accounted for this disparity of reported drug use (3). Several reasons may explain the discrepancy between those results and the present study's findings. First, Delaney et al. excluded patients with cardiovascular disease, which represented a large number of individuals in our study. Second, pharmaceutical policies and reimbursement rules in Quebec differ substantially from those of the United States where these studies were undertaken. As ASA is paid for when the drug is prescribed, there was little financial incentive for our study's individuals to buy this drug OTC. Moreover, elderly individuals who use ASA for cardiovascular prevention must purchase several other prescribed drugs as well. Therefore, many elderly individuals reach their cap on the monthly contributions required under their drug plan and filling their ASA prescriptions may be less costly than buying the drugs OTC.

We observed that only 2 out of 8 participants who reported using ASA without a prescription were using the drug as a cardio-protective agent. This finding contrasts with the results of a population-based US survey of 3128 adults in 2000-2002 (9). Among 164 participants who reported using nonprescription ASA specifically for perceived cardiovascular health benefits, nearly half $(76 / 164)$ of them had a medical prescriber advise or recommend ASA.

Our results have important implications for studies that use administrative databases to investigate ASA use. Prescribed drugs that are reimbursed under the Quebec drug plan, such as ASA, will be included in the Quebec prescription claims database. A previous validation study found that the prescription claims database in Quebec is accurate (10). Because the vast majority of elderly individuals are insured under the drug plan (11), all ASA prescriptions registered under pharmacy records also should be included in the prescription claims database. Therefore, in contrast with other databases, in which using prescription claims may lead to misleading estimates of ASA exposure $(2,3,12)$, researchers may feel confident about the slight potential for exposure misclassification for the cardiovascular use of ASA within the Quebec's drug claims database for elderly people. Our results may also hold true for other provinces in Canada that share a similar pharmaceutical reimbursement scheme, although more studies are needed to confirm this hypothesis. Nonetheless, we acknowledge that there is a short-term and random misclassification of ASA exposure when the drug is used for analgesia by individuals who do not use the drug as a cardio-protective agent. However, based on our results, such misclassification would only affect a small number of individuals in populations of elderly individuals in Quebec.

There are some limitations to this study. First, this study used a relatively small sample size of participants. Second, our study was limited to elderly patients who were recruited in the Quebec City area and volunteered to participate. Important population characteristics, such as age and socio-economic status, were similar to those of Quebec's elderly population, but our study sample may differ from those of other populations. Therefore, our conclusions may not be generalizable to dissimilar populations or settings. Further studies should investigate whether ASA use differs according to age or drug plan coverage.

In conclusion, our results show that a large proportion of elderly patients in the province of Quebec fill their prescription when physicians prescribe ASA to them. Pharmacy records and prescription claims databases are thus reliable sources of information on prescribed ASA use in Quebec.

\section{ACKNOWLEDGMENTS}

This study was funded by the Réseau québécois de recherche sur l'utilisation des médicaments 
and by the Axe santé mentale du Réseau québécois de recherche sur le vieillissement. We wish to thank Michel Gaudet, MSc, for assistance in data analysis. We also express our appreciation to Jocelyne Moisan, $\mathrm{PhD}$, for her valuable input and contributions. Caroline Sirois is supported by a Fonds de la recherche en santé du Québec scholarship. The authors declare no financial, personal, commercial, or academic conflicts of interest, and no conflicts related to study interpretation.

\section{REFERENCES}

1. Lau H, de Boer A, Beuning $K$, Porsius A. Validation of pharmacy records in drug exposure assessment. J Clin Epidemiol, 1997;50:619-625.

2. Delaney J, Biggs M, Kronmal R, Psaty B. Demographic, medical, and behavioral characteristics associated with over the counter non-steroidal anti-inflammatory drug use in a population-based cohort: results from the MultiEthnic Study of Atherosclerosis. Pharmacoepidemiol Drug Saf, 2011;20:83-89. DOI: $10.1002 /$ pds.2065.

3. Ilkhanoff L, Lewis J, Hennessy S, Berlin J, Kimmel S. Potential limitations of electronic database studies of prescription non-aspirin nonsteroidal anti-inflammatory drugs (NANSAIDs) and risk of myocardial infarction (MI). Pharmacoepidemiol Drug Saf, 2005;14:513-522.

4. Guénette L, Moisan J, Préville M, Boyer R. Measures of adherence based on self-report exhibited poor agreement with those based on pharmacy records. J Clin Epidemiol, 2005;58:924933.

5. Guénette L, Moisan J. Elderly people's knowledge of the purpose of their medicines. Am J Geriatr Pharmacother, 2011;9:49-57.

6. WHO Collaborating Center for Drug Statistics Methodology. Anatomical Therapeutic Chemical (ATC) Classification Index. Oslo; 2007.

7. Fleiss J. Statistical methods for rates and proportions. Second ed. New York: John Wiley \& Sons, Inc. 1981.

8. Landis J, Koch G. The measurement of observer agreement for categorical data. Biometrics, 1977;33:159-174.

9. Artz M, Harnack L, Duval S, Armstrong C, Arnett $\mathrm{D}$, Luepker R. Use of nonprescription medications for perceived cardiovascular health. Am J Prev Med, 2006;30:78-81.

10. Tamblyn R, Lavoie G, Petrella L, Monette J. The use of prescription claims databases in pharmacoepidemiological research: The accuracy and comprehensiveness of the prescription claims database in Quebec. $J$ Clin Epidemiol, 1995;48:999-1009.

11. Régie de l'assurance maladie du Québec. Rapport annuel de gestion 2009-2010. 2010.

12. Garg R, Glazer N, Wiggins K, Newton K, Thacker E, Smith N, Siscovick DS, Psaty, BM, Heckbert SR. Ascertainment of warfarin and aspirin use by medical record review compared with automated pharmacy data. Pharmacoepidemiol Drug Saf, 2011;20:313-316. DOI: 10.1002/pds.2041. 